\title{
Multiplicities of discriminants
}

\author{
PaOlo Aluffi ${ }^{1}$ \\ FERnANDo CUKIERMAN
}

\begin{abstract}
We compute the multiplicity of the discriminant of a line bundle $\mathcal{L}$ over a nonsingular variety $S$ at a given section $X$, in terms of the Chern classes of $\mathcal{L}$ and of the cotangent bundle of $S$, and the Segre classes of the jacobian scheme of $X$ in $S$. For $S$ a surface, we obtain a precise formula that expresses the multiplicity as a sum of a term due to the non-reduced components of the section, and a term that depends on the Milnor numbers of the singularities of $X_{\text {red }}$. Also, under certain hypotheses, we provide formulas for the "higher discriminants" that parametrize sections with a singular point of prescribed multiplicity. As an application, we obtain criteria for the various discriminants to be "small".
\end{abstract}

\section{INTRODUCTION.}

Let $S \subset \mathbb{P}^{N}$ be a smooth variety over an algebraically closed field of characteristic 0 . Denote by $D \subset \check{\mathbb{P}}^{N}$ the dual variety of $S$, consisting of all the hyperplanes in $\mathbb{P}^{N}$ that are tangent to $S$. The basic question that we consider in this note is: if $X \in D$ is a singular hyperplane section of $S$, what is the multiplicity $m_{X} D$ of $D$ at $X ?$

Our answer is in the style of the well known formula for the degree of the dual in terms of the Chern classes of $S$ and of the hyperplane bundle $\mathcal{L}$ (see for example $[3]$, p. 63). We associate with each hyperplane section $X$ a zero-dimensional class in the Chow group of $S$, obtained by capping the Segre class in $S$ of the singular scheme of $X$ by the Chern classes of the bundle $P^{1} \mathcal{L}$ of principal parts of order one (which are easily expressible in terms of the classes of $\mathcal{L}$ and of the cotangent bundle of $S$ ). Assuming that $D$ is a hypersurface, the multiplicity of $D$ at $X$ is then essentially the degree of this class. For example, if $S=\mathbb{P}^{2}$, and one writes the section $X$ as $\sum_{i} m_{i} X_{i}$ with $m_{i}$ natural numbers and $X_{i}$ irreducible divisors, then $m_{X}(D)$ has a simple expression in terms of the integers $m_{i}$, the degrees of the $X_{i}$, and the singularities of $X_{\text {red }}=\sum_{i} X_{i}$ (see 3.1).

The main general formula is stated in $\S 1$, in a more general setting also addressing the same question for the 'higher discriminants' $D^{(r)} \subset \check{\mathbb{P}}^{N}$, consisting of sections of $S$ that have a point of multiplicity at least $r+1$. The formula gives $m_{X} D^{(r)}$ under a hypothesis on the dimension of the osculating planes of $S$ (automatic in the case of dual varieties) and assuming that the discriminants have the expected dimension. In these hypotheses, the formula also specializes easily to compute the degree of the discriminants (Corollary 1.1).

In fact the class introduced in $\S 1$ can be used to detect whether the dual variety is not a hypersurface - that is, whether it is 'small': we show (Proposition 1.3) that the class vanishes if and only if the dual is small - again, we prove the corresponding result for all discriminants, under the same hypothesis on the osculating planes.

The main difficulty in applying the theorem to specific situations lies in the computation of the Segre class $s\left(J^{r} X, S\right)$ of the Jacobian schemes of a hyperplane section. One important case occurs when $S$ is a surface, or more generally when the

\footnotetext{
${ }^{1}$ Supported in part by the Max-Planck-Institut für Mathematik
} 
singularities of $X_{\text {red }}$ are isolated: then one can write the Segre class - and therefore the multiplicity of $D^{(r)}$ at $X$-as the sum of an easily computable term and a contribution $\mu_{r}$ due to the singularities of $X_{\text {red }}$. The result is stated in Corollary 1.2 for all discriminants. The rôle of $\mu_{1}$ is clarified in $\S 2$, where we discuss the dual of a surface using Lefschetz pencils and show (Proposition 2.1) that in this case the contribution $\mu_{1}$ is the sum of the Milnor numbers of the singularities of $X_{\text {red }}$. This particular case was our main original motivation; $\S 2$ may be read independently of the other sections.

In $\S 3$ we provide several examples of explicit computations of multiplicities. Also, we give a simple proof of a known criterion for the product of projective spaces $\mathbb{P}^{n_{1}} \times \cdots \times \mathbb{P}^{n_{r}}$ to have small dual under the Segre embedding (Example 3.5).

Another approach to the computation of the multiplicity of a dual variety can be found in $[\mathbf{1 2}]$, generalizing earlier results $([\mathbf{1}],[\mathbf{1 1}])$ : there the multiplicity is written in terms of generalized Milnor numbers associated with the singularities of the section and of its intersections with general linear subspaces.

Acknowledgements. We thank Joe Harris who asked us an interesting question that originated this work. Also, we are grateful to Piotr Pragacz for useful conversations. The first named author wishes to thank the Max-Planck-Institut für Mathematik for the support and the hospitality during part of the preparation of this note. An earlier version of this work appeared as MPI preprint 92/21.

\section{$\S 1$. Multiplicities OF Discriminants.}

Let $\mathcal{L}$ be a line bundle on a smooth $n$-dimensional variety $S$, and fix a vector space $V$ mapping linearly to $H^{0}(S, \mathcal{L})$. If $X \in \mathbb{P} V$ and some representative of $X$ maps to a section $s \neq 0$ of $\mathcal{L}$, then we can associate with $X$ the divisor of zeros of $s$. We say that $X$ 'has multiplicity $>r$ ' at a point $p$ of $S$ if the corresponding divisor does. More precisely, let $P^{r} \mathcal{L}$ denote the $r$-th bundle of principal parts of $\mathcal{L}$ (see [8], [13]). We recall that $P^{0} \mathcal{L}=\mathcal{L}$ and for $r \geq 0, P^{r} \mathcal{L}$ is locally free of rank $\left(\begin{array}{c}r+n \\ n\end{array}\right)$. One has natural morphisms of locally free sheaves

$$
t_{r}: S \times H^{0}(S, \mathcal{L}) \rightarrow P^{r} \mathcal{L}
$$

that can be thought of as 'truncated Taylor expansion' maps. Then $X$ has multiplicity $>r$ at $p$ if $t_{r}(p)(s)=0$. We will abuse notations and denote by $X$ the divisor of $S$ determined by $X \in \mathbb{P} V$; or $S$ itself if all representatives of $X$ map to the zero-section (observe that in this latter case $X$ has multiplicity $>r$ for all $r$ ).

For $r \geq 0$ we define the ' $r$-th discriminant $D^{(r)}$ of $\mathcal{L}$ ' by

$$
D^{(r)}=\{X \in \mathbb{P} V \quad \text { s.t. } X \text { has a point of multiplicity }>r\} .
$$

Thus $D^{(0)}=\mathbb{P} V$, and $D^{(1)}$ is the ordinary discriminant in $\mathbb{P} V$; if $(V, \mathcal{L})$ is very ample, then $D^{(1)}$ is the dual variety of $S \subset \mathbb{P} V^{\vee}$. Given a specific $X \in \mathbb{P} V$, we aim to computing the multiplicity $m_{X} D^{(r)}$ of the $r$-th discriminant at $X$.

We shall denote by $\mathcal{V}$ the trivial bundle $S \times V$, and by $\alpha_{r}$ the composition

$$
\alpha_{r}: \mathcal{V} \rightarrow S \times H^{0}(S, \mathcal{L}) \stackrel{t_{r}}{\longrightarrow} P^{r} \mathcal{L}
$$

The ' $r$-th osculating plane' of $S$ at $p \in S$ in $\mathbb{P} V^{\vee}$ will be the linear subspace of $\mathbb{P} V^{\vee}$ defined by the fiber of the kernel of $\alpha_{r}$ over $p$. For example, if $(V, \mathcal{L})$ is very ample 
then the first osculating plane to $S$ at $p$ is the tangent space to $S$ at $p$. Comparing ranks, we observe that the dimension of the $r$-th osculating plane at any $p \in S$ is necessarily bounded by the minimum of $\operatorname{dim} \mathbb{P} V$ and $\left(\begin{array}{c}r+n \\ n\end{array}\right)-1$.

Also, for $X \in \mathbb{P} V$ corresponding to a section $s$ of $\mathcal{L}$, let $J^{r} X$ denote the scheme of zeros of $t_{r}(s)$ (that is, the subscheme defined locally by all derivatives up to order $r$ of $s$ ): thus $J^{1} X$ is the ordinary Jacobian scheme of $X$, supported on its singularities; $J^{2} X$ is supported on the subset of $S$ along which $X$ has multiplicity $>2$, and so on.

Finally, let $\gamma_{i}$ be the degree (in the sense of [3], Definition 1.4) $\operatorname{deg}\left[J^{i} X\right]$ of the cycle $\left[J^{i} X\right]$ for the general element $X \in D^{(i)}$. So in particular $\gamma_{i}=0$ if the set of points along which the general $X$ has multiplicity $>i$ has positive (pure) dimension. $\gamma_{i}=1$ if the general element of $D^{(i)}$ has exactly one point of multiplicity $i+1$ : for example this is the case for $i=1$ (in char. 0$)$ if $(V, \mathcal{L})$ is very ample and the dual of $X$ is a hypersurface $([\mathbf{9}])$.

ThEOREM. With notations as above, suppose that at each $p \in S$ the $r$-th osculating plane has dimension $\left(\begin{array}{c}n+r \\ n\end{array}\right)-1$; also, denote by $\Omega$ the cotangent bundle of $S$. Then for $0<i \leq r$

$$
\gamma_{i} \cdot m_{X} D^{(i)}=\operatorname{deg}\left\{c\left(\operatorname{Sym}^{i}(\Omega \oplus \mathcal{O}) \otimes \mathcal{L}\right) \cap s\left(J^{i} X, S\right)\right\}_{0} .
$$

(Here and in the following, $c(\cdot), s(\cdot)$ denote resp. Chern and Segre classes, in the sense of $[\mathbf{3}] ;\{\cdot\}_{0}$ denotes the component of dimension 0 of the class between brackets)

REMARKS. The condition on the dimension of the osculating planes amounts to requiring that the map $\alpha_{r}: \mathcal{V} \rightarrow P^{r} \mathcal{L}$ be surjective. $\mathcal{V}$ surjects onto $P^{0} \mathcal{L}=\mathcal{L}$ when the natural map $S \rightarrow \mathbb{P} V^{\vee}$ has no base locus; $\mathcal{V}$ surjects onto $P^{1} \mathcal{L}$ if the same map is locally a closed immersion. For higher $i$, this surjectivity assumption is 'reasonable' if the image of $S$ in $\mathbb{P} V^{\vee}$ is nondegenerate and $n+\left(\begin{array}{c}r+n \\ n\end{array}\right) \leq \operatorname{dim} V$. The assumption may still hold beyond this bound, but then severe restrictions on $S$ and $\mathcal{L}$ come into effect. For example, it is shown in [4] that if $\left(\begin{array}{c}n+r \\ n\end{array}\right)=\operatorname{dim} V$ and the surjectivity condition is satisfied, then the image of $S$ in $\mathbb{P} V^{\vee}$ is necessarily the $r$-th Veronese embedding of $\mathbb{P}^{n}$.

Applying the theorem to $V \oplus 1$, with $X$ the section corresponding to the second summand, gives

COROLlary 1.1. In the hypotheses of the theorem,

$$
\gamma_{i} \cdot \operatorname{deg} D^{(i)}=\operatorname{deg}\left\{c\left(\operatorname{Sym}^{i}(\Omega \oplus \mathcal{O}) \otimes \mathcal{L}\right) \cap[S]\right\}_{0}
$$

(indeed, the discriminant in $\mathbb{P}(V \oplus 1)$ is the cone over the discriminant in $\mathbb{P} V$, with vertex $X$.) For $i=1$, this is equivalent to the computation of the degree of the dual variety in $[\mathbf{3}]$, p. 63 .

Next, denote by $X^{(r)}$ the cycle of codimension 1 in $S$ on which $J^{r} X$ is supported (so e.g., $X^{(r)}=0$ if $J^{r} X$ has no components of codimension 1 in $S$ ). That is, if $X=\sum \alpha_{i} X_{i}$ with $X_{i}$ irreducible divisors, then $X^{(r)}=\sum\left(\alpha_{i}-r\right) X_{i}$, the sum extended over the $i$ 's such that $\alpha_{i}>r$. Abusing notations, we write $X$ for $X^{(0)}$; 
thus $X^{(1)}=X-X_{\text {red }}$, and $X^{(r)}$ is the $r$-th iteration of this operation. Observe that $J^{r} X$ and $X^{(r)}$ coincide away from the singular locus of $X_{\text {red. }}$.

Now if $X_{\text {red }}$ has only isolated singularities, then with this notation and using [3], Prop. 9.2, one gets:

$$
s\left(J^{r} X, S\right)=s\left(X^{(r)}, S\right)+\mu_{r}=\sum_{j=1}^{d}(-1)^{j+1} X^{(r)^{j}}+\mu_{r}
$$

where $\mu_{r}$ is a contribution (in dimension 0) supported on the singularities of $X_{\text {red }}$ : more precisely, $\mu_{r}$ is the Segre class in $S$ of the residual scheme to $X^{(r)}$ in $J^{r} X$. Note that only singular points of $X_{\text {red }}$ at which $X$ has multiplicity $>r$ contribute to $\mu_{r}$.

In the case when $S$ is a surface, writing out $c_{1}\left(\operatorname{Sym}^{i}(\Omega \oplus \mathcal{O}) \otimes \mathcal{L}\right)$ and using the above observation gives:

Corollary 1.2. Let $S$ be a smooth surface. With the above notations, suppose that at each $p \in S$ the $r$-th osculating plane has dimension $\left(\begin{array}{c}r+2 \\ 2\end{array}\right)-1$; and let $K$ denote the canonical divisor of $S$. Then for $0<i \leq r$

$$
\gamma_{i} \cdot m_{X} D^{(i)}=\left[\left(\begin{array}{c}
i+2 \\
2
\end{array}\right) X-X^{(i)}+\left(\begin{array}{c}
i+2 \\
3
\end{array}\right) K\right] \cdot X^{(i)}+\mu_{i}
$$

In $\S 2$ we will approach this case over $\mathbb{C}$ from a different angle; and comparing the formula we will obtain with this result it follows that in fact $\mu_{1}$ is the sum of the Milnor numbers of the singularities of $X_{\text {red }}$ when $S$ is a surface. It would be interesting to have a similar interpretation for the contributions $\mu_{i}, i>1$.

PROOF OF THE THEOREM: The proof is a straightforward application of the theory of Segre classes and multiplicities, as developed in [3], Chapter 4.

Thinking of $X$ as a point of $D^{(i)}$, one has $s\left(X, D^{(i)}\right)=m_{X} D^{(i)} X([\mathbf{3}], 4.3)$ : thus $m_{X} D^{(i)}=\operatorname{deg} s\left(X, D^{(i)}\right)$. Now we see $D^{(i)} \subset \mathbb{P} V$ as the projection from $S \times \mathbb{P} V=\mathbb{P} \mathcal{V}$ of the correspondence

$$
\mathcal{D}^{(i)}=\{(p, X) \in \mathbb{P} \mathcal{V} \text { s.t. } X \text { has multiplicity }>i \text { at } p\} .
$$

In other words, $\mathcal{D}^{(i)}=\mathbb{P} \mathcal{N}_{i}$, where $\mathcal{N}_{i}$ is the kernel of the 'truncated Taylor map' $\mathcal{V} \rightarrow P^{i} \mathcal{L}$. Also, observe that by identifying the fiber of $\mathbb{P} \mathcal{V}$ over $X$ with $S$, the fiber of $\mathcal{D}^{(i)}$ is identified with $J^{i} X$; and the number $\gamma_{i}$ of the statement of the theorem is the degree of the projection $\mathcal{D}^{(i)} \rightarrow D^{(i)}$.

If $\pi$ denotes the projection $\mathcal{D}^{(i)} \rightarrow D^{(i)}$, then Proposition 4.2 (a) from [3] and the above observations give $\gamma_{i} \cdot s\left(X, D^{(i)}\right)=\pi_{*} s\left(J^{i} X, \mathcal{D}^{(i)}\right)$; taking degrees, we get

$$
\gamma_{i} \cdot m_{X} D^{(i)}=\operatorname{deg} s\left(J^{i} X, \mathcal{D}^{(i)}\right)
$$

So we are after this latter Segre class. Now both $S$ and $\mathcal{D}^{(i)}\left(=\mathbb{P} \mathcal{N}_{i}\right)$ are nonsingular, so [3], 4.2.6 gives

$$
c\left(T \mathcal{D}^{(i)}\right) \cap s\left(J^{i} X, \mathcal{D}^{(i)}\right)=c(T S) \cap s\left(J^{i} X, S\right)
$$


from which

$$
s\left(J^{i} X, \mathcal{D}^{(i)}\right)=c\left(T \mathcal{D}^{(i)} \mid S\right)^{-1} \cap s\left(J^{i} X, S\right)
$$

where $T \mathcal{D}^{(i)} \mid S$ is the relative tangent bundle of $\mathcal{D}^{(i)}$ over $S$. Its classes are computed by using the Euler sequence for $\mathcal{D}^{(i)}=\mathbb{P} \mathcal{N}_{i}$; since the restriction of $\mathcal{O}_{\mathbb{P} \mathcal{N}_{i}}(1)$ to the fiber over $X$ is trivial, we get

$$
s\left(J^{i} X, \mathcal{D}^{(i)}\right)=c\left(\mathcal{N}_{i}\right)^{-1} \cap s\left(J^{i} X, S\right) .
$$

To finish off we just have to show that $c\left(\mathcal{N}_{i}\right)^{-1}=c\left(\operatorname{Sym}^{i}(\Omega \oplus \mathcal{O}) \otimes \mathcal{L}\right)$ for $0<i \leq r$, in the hypotheses of the theorem. But if $\mathcal{V} \rightarrow P^{r} \mathcal{L}$ is surjective, then following with the truncation surjections $P^{i} \mathcal{L} \rightarrow P^{i-1} \mathcal{L}$ shows that $\alpha_{i}: \mathcal{V} \rightarrow P^{i} \mathcal{L}$ is surjective for $0 \leq i \leq r$ : thus $c\left(\mathcal{N}_{i}\right)^{-1}=c\left(P^{i} \mathcal{L}\right)$ since $\mathcal{N}_{i}=\operatorname{ker}\left(\alpha_{i}\right)$.

Now use the standard exact sequence for the bundles of principal parts:

$$
0 \rightarrow \operatorname{Sym}^{k} \Omega \otimes \mathcal{L} \rightarrow P^{k} \mathcal{L} \rightarrow P^{k-1} \mathcal{L} \rightarrow 0 \quad .
$$

Recalling $P^{0} \mathcal{L}=\mathcal{L}$, this gives at once

$$
c\left(P^{i} \mathcal{L}\right)=c(\mathcal{L}) c(\Omega \otimes \mathcal{L}) c\left(\operatorname{Sym}^{2} \Omega \otimes \mathcal{L}\right) \cdots c\left(\operatorname{Sym}^{i} \Omega \otimes \mathcal{L}\right) \quad:
$$

and this equals $c\left(\operatorname{Sym}^{i}(\Omega \oplus \mathcal{O}) \otimes \mathcal{L}\right)$, as stated.

In a different vein, we can derive from the theorem a criterion for the $r$-th discriminant to be "small". Under the surjectivity of $\alpha_{r}$, it follows easily that the codimension of $D^{(r)}$ in $\mathbb{P} V$ is $\geq\left(\begin{array}{c}r+n \\ n\end{array}\right)-n$. We say that the $r$-th discriminant is small if its codimension in $\mathbb{P} V$ is $>\left(\begin{array}{c}r+n \\ n\end{array}\right)-n$.

Proposition 1.3. Suppose that at each $p \in S$ the $r$-th osculating plane has dimension $\left(\begin{array}{c}n+r \\ n\end{array}\right)-1$. Then the following are equivalent:

(1) For all $X \in \mathbb{P} V$

$$
\left\{c\left(\operatorname{Sym}^{r}(\Omega \oplus \mathcal{O}) \otimes \mathcal{L}\right) \cap s\left(J^{r} X, S\right)\right\}_{0}=0 \quad ;
$$

(2) For some $X \in D^{(r)}$

$$
\operatorname{deg}\left\{c\left(\operatorname{Sym}^{r}(\Omega \oplus \mathcal{O}) \otimes \mathcal{L}\right) \cap s\left(J^{r} X, S\right)\right\}_{0}=0 \quad ;
$$

$$
\operatorname{deg}\left\{c\left(\operatorname{Sym}^{r}(\Omega \oplus \mathcal{O}) \otimes \mathcal{L}\right) \cap[S]\right\}_{0}=0 \quad ;
$$

(4) the $r$-th discriminant $D^{(r)}$ is small.

Proof: The implications (1) $\Longrightarrow(2) \Longrightarrow(4)$ and (3) $\Longleftrightarrow(4)$ are immediate, in view of the theorem and Corollary 1.1.

Thus we just have to show that (4) implies (1), that is that if the projection $\pi: \mathcal{D}^{(r)}=\mathbb{P} \mathcal{N}_{r} \rightarrow D^{(r)}$ is not generically finite, then the class in (1) vanishes for all $X \in D^{(r)}$ (the class vanishes automatically if $X \notin D^{(r)}$ ). Observe that the theorem implies immediately that the degree of the class is 0 if (4) holds, since in this case $\gamma_{r}=0$; proving that the class itself is 0 requires a little more work.

Recall from the proof of the theorem that the class between brackets in (1) equals the Segre class $s\left(J^{r} X, \mathcal{D}^{(r)}\right)$, and that $J^{r} X=\pi^{-1}(X)$. Thus we have to show that if $\pi$ is not generically finite then $\left\{s\left(\pi^{-1}(X), \mathcal{D}^{(r)}\right)\right\}_{0}=0$ (notice that this follows immediately from [3], Proposition 4.2 (b) if $\pi$ is flat over a neighborhood of $X$ ). This is a consequence of the following general remark. 
Lemma. Let $\pi: Y^{\prime} \rightarrow Y$ be an onto morphism of irreducible schemes, with $\operatorname{dim} Y^{\prime}>\operatorname{dim} Y$; let $X \in Y$ be a closed point, and denote by $X^{\prime}=\pi^{-1}(X)$ the inverse image scheme. Then $\left\{s\left(X^{\prime}, Y^{\prime}\right)\right\}_{0}=0$.

Proof: Let $C, C^{\prime}$ resp. denote the normal cones to $X$ in $Y$, and $X^{\prime}$ to $Y^{\prime}$ resp. In this set up we get (see [3], proof of Prop. 4.2) an induced morphism

$$
\mathbb{P}\left(C^{\prime} \oplus 1\right) \stackrel{G}{\rightarrow} \mathbb{P}(C \oplus 1),
$$

such that the canonical line bundle on $\mathbb{P}\left(C^{\prime} \oplus 1\right)$ is the pull-back $G^{*} \mathcal{O}(1)$ of the canonical line bundle on $\mathbb{P}(C \oplus 1)$. If $q^{\prime}$ denotes the projection from $\mathbb{P}\left(C^{\prime} \oplus 1\right)$ to $X^{\prime}$, then

$$
\left\{s\left(X^{\prime}, Y^{\prime}\right)\right\}_{0}=q_{*}^{\prime}\left(c_{1}\left(G^{*} \mathcal{O}(1)\right)^{\operatorname{dim} Y^{\prime}} \cap\left[\mathbb{P}\left(C^{\prime} \oplus 1\right)\right]\right) ;
$$

but this is necessarily 0: indeed, certainly there exist $\operatorname{dim} Y^{\prime}$ sections of $\mathcal{O}(1)$ which don't vanish simultaneously anywhere on $\mathbb{P}(C \oplus 1)$ (because $\operatorname{dim} Y<\operatorname{dim} Y^{\prime}$ ), and these pull-back to $\operatorname{dim} Y^{\prime}$ sections of $G^{*}(\mathcal{O}(1))$ that don't vanish simultaneously anywhere on $\mathbb{P}\left(C^{\prime} \oplus 1\right)$.

This proves the Lemma, and concludes the proof of the proposition.

REMARKS. If the $D^{(r)}$ is small then not only the degree, but the class itself in (3) must vanish (by (1), cf. the argument for Corollary 1.1).

We find the implications $(3) \Longrightarrow(1),(2) \Longrightarrow(1)$ rather striking, as they impose in the hypotheses of the theorem a strong condition on the Segre classes of the Jacobian schemes of divisors. For example, the vanishing of the class for one singular hyperplane section of a smooth variety $S \subset \mathbb{P}^{n}$ (in fact, the vanishing of its degree suffices) implies the vanishing of the class for all hyperplane sections, and that the dual of $S$ is small. See Example 3.5 for an illustration of this fact.

\section{THE CASE $\operatorname{dim}(S)=2$.}

In this section we give an independent derivation of Corollary 1.2 for $i=1$, over the complex numbers. In this particular situation we obtain more precise information, namely, that $\mu_{1}$ is the sum of the Milnor numbers of the singularities of $X_{\text {red }}$.

Let $S$ be a smooth compact algebraic surface over the complex numbers, $|\mathcal{L}|$ a very ample complete linear system on $S$ and $D=D^{(1)} \subset|\mathcal{L}|$ the discriminant hypersurface, consisting of singular members of $|\mathcal{L}|$. It is known [2] that the dual variety $D$ is actually a hypersurface.

Our goal is to determine, for each $X \in|\mathcal{L}|$, the multiplicity $m_{X}(D)$ of the hypersurface $D$ at the point $X$. Suppose that

$$
X=\sum_{1 \leq i \leq r} n_{i} X_{i}
$$

where $X_{i}$ is reduced and irreducible. Take a general $Y \in|\mathcal{L}|$ (i.e., a $Y$ intersecting $X_{\text {red }}=\sum_{1 \leq i \leq r} X_{i}$ transversally) and denote by $L \subset|\mathcal{L}|$ the pencil containing $X$ and $Y$. Then

$$
m_{X}(D)=\operatorname{deg}(D)-s
$$


where $s$ is the number of singular members of $L$ different from $X$ (each of these singular members has one node as singular set). In order to determine $s$ we shall blow up $S$ to construct a family parametrized by $L$ and use Lefschetz' formula ([7], page 509).

For each $1 \leq i \leq r$, denote by $p_{i j}\left(1 \leq j \leq Y \cdot X_{i}\right)$ the points of intersection of $Y$ and $X_{i}$. Let $\widehat{S}$ denote the surface obtained from $S$ by blowing up at each $p_{i j} n_{i}$ times (in the direction of $Y$ ). The induced pencil on $\widehat{S}$ is base-point-free and gives a map $f: \widehat{S} \rightarrow \mathbb{P}^{1}$. If $E_{i j}^{k}, k=1, \ldots, n_{i}$ are the exceptional divisors at $p_{i j}$ then the fiber of $f$ at the point 0 corresponding to $X$ is

$$
f^{*}(0)=X+\sum_{i, j} \sum_{1 \leq k \leq n_{i}-1}\left(n_{i}-k\right) E_{i j}^{k}
$$

In other words, the special fiber is isomorphic to $X$ with strings of $\mathbb{P}^{1}$ 's (each $\mathbb{P}^{1}$ with a certain multiplicity) attached at the points $p_{i j}$; each string has $n_{i}-1$ components. We now denote

$$
X^{\prime}=\left(f^{*}(0)\right)_{\mathrm{red}}=X_{\mathrm{red}}+\sum_{i, j} T_{i j}
$$

the reduced (i.e., set-theoretic) fiber of $f$ at 0 , where $T_{i j}=\sum_{1 \leq k \leq n_{i}-1} E_{i j}^{k}$ is the (reduced) string attached at $p_{i j}$. The argument in [7] works in the present circumstance (the topology does not "see" the multiplicities of the special fiber) and gives

$$
\chi(S)=2 \chi(Y)-Y \cdot Y+\left(\chi\left(X^{\prime}\right)-\chi(Y)\right)+\sum_{1 \leq \lambda \leq s}\left(\chi\left(Y_{\lambda}\right)-\chi(Y)\right)
$$

where $\chi$ denotes topological Euler characteristic and $Y_{\lambda}$ are the singular fibers for $\lambda \neq 0$. Since $\chi\left(Y_{\lambda}\right)-\chi(Y)=1([\mathbf{7}]$ or $(5)$ below $)$ and $\operatorname{deg}(D)=\chi(S)-2 \chi(Y)+Y . Y$ ([7] or Corollary (1.1)), combining with (2) we obtain

$$
m_{X}(D)=\chi\left(X^{\prime}\right)-\chi(Y)
$$

In order to compute $\chi\left(X^{\prime}\right)$, denoting $T=\bigcup_{i, j} T_{i j}$ we have

$$
\begin{aligned}
\chi\left(X^{\prime}\right) & =\chi\left(X_{\text {red }} \cup T\right)=\chi\left(X_{\text {red }}\right)+\chi(T)-\chi\left(X_{\text {red }} \cap T\right) \\
& =\chi\left(X_{\text {red }}\right)+\sum_{i, j} \chi\left(T_{i j}\right)-\sum_{i, j} \chi\left(\left\{p_{i j}\right\}\right)=\chi\left(X_{\text {red }}\right)+\sum_{i, j} n_{i}-\sum_{i, j} 1 \\
& =\chi\left(X_{\text {red }}\right)+X \cdot\left(X-X_{\text {red }}\right)
\end{aligned}
$$

Now we compute $\chi\left(X_{\text {red }}\right)$. Let $Z=\sum_{1 \leq i \leq r} Z_{i}$ be a reduced (connected, for simplicity) curve with normalization

$$
\rho: \widetilde{Z}=\coprod_{1 \leq i \leq r} \widetilde{Z}_{i} \rightarrow Z
$$

If $p \in Z$ is a singular point, denote $B(p)=\rho^{-1}(p)$ the set of branches of $Z$ at $p$. Topologically, $Z$ is obtained from the smooth surface $\widetilde{Z}$ by identifying each of the 
sets $B(p)$ to a point $p$. Recall from $[6]$, page 96 , that if $X$ is a topological space and $A \subset X$ is a subspace such that $(X, A)$ is a collared pair then $\chi^{\#}(X)-\chi^{\#}(X / A)=$ $\chi^{\#}(A)$, where $X / A$ is the space obtained from $X$ by identifying $A$ to a point and $\chi^{\#}$ is Euler characteristic for augmented homology. If $X$ is a manifold and $A$ consists of $a$ points then $\chi^{\#}(X)-\chi^{\#}(X / A)=\chi(X)-\chi(X / A)=a-1$. Applying this for each singular point we obtain

$$
\chi(\widetilde{Z})-\chi(Z)=\sum_{p \in Z}(b(Z, p)-1)
$$

where $b(Z, p)$ is the number of branches of $Z$ at $p$. Also, from the exact sequence of sheaves

$$
0 \rightarrow \mathcal{O}_{Z} \rightarrow \rho_{*} \mathcal{O}_{\widetilde{Z}} \rightarrow \rho_{*} \mathcal{O}_{\widetilde{Z}} / \mathcal{O}_{Z} \rightarrow 0
$$

we obtain $1-r+\sum_{p \in Z} \delta(Z, p)-h^{1}\left(\mathcal{O}_{Z}\right)+h^{1}\left(\mathcal{O}_{\widetilde{Z}}\right)=0$, where we let $\delta(Z, p)=$ $\operatorname{length}_{p}\left(\rho_{*} \mathcal{O}_{\widetilde{Z}} / \mathcal{O}_{Z}\right)$, and then, combining with (5),

$$
\chi(Z)=2-2 p_{a}(Z)+\sum_{p \in Z} \mu(Z, p)
$$

where $\mu(Z, p)=2 \delta(Z, p)-b(Z, p)+1$ is the Milnor number of $(Z, p)$. Combining $(3),(4)$ and (6) we obtain

$$
\begin{aligned}
& m_{X}(D)=\chi\left(X_{\text {red }}\right)-\chi(Y)+X \cdot\left(X-X_{\text {red }}\right) \\
& \quad=\left(2-2 p_{a}\left(X_{\text {red }}\right)\right)-\left(2-2 p_{a}(Y)\right)+X \cdot\left(X-X_{\text {red }}\right)+\sum_{p \in X_{\text {red }}} \mu\left(X_{\text {red }}, p\right) \\
& \quad=\left(K_{S}+Y\right) \cdot Y-\left(K_{S}+X_{\text {red }}\right) \cdot X_{\text {red }}+X \cdot\left(X-X_{\text {red }}\right)+\sum_{p \in X_{\text {red }}} \mu\left(X_{\text {red }}, p\right)
\end{aligned}
$$

and rearranging we finally obtain

Proposition 2.1. If $S$ is a smooth compact algebraic surface over the complex numbers then -with notation as above- for any $X \in D$ we have

$$
m_{X}(D)=\left(X-X_{\mathrm{red}}\right) \cdot\left(K_{S}+2 X+X_{\mathrm{red}}\right)+\sum_{p \in X_{\mathrm{red}}} \mu\left(X_{\mathrm{red}}, p\right)
$$

Comparing with Corollary $1.2, i=1$, yields $\mu_{1}=\sum_{p \in X_{\text {red }}} \mu\left(X_{\text {red }}, p\right)$ as claimed at the beginning of this section.

\section{EXAMPLES.}

In this section we apply the results obtained thus far to a few concrete situations, to illustrate the actual 'computability' of the formulas.

Example 3.1. Applying the result in $\S 2$ we may compute the multiplicity of the discriminant of the space of plane curves of a given degree $d$ at a singular curve $X$. If $d^{(1)}$ is the degree of $X^{(1)}=X-X_{\text {red }}$, then Proposition 2.1 gives

$$
m_{X} D=\left[3(d-1)-d^{(1)}\right] d^{(1)}+\mu
$$


where $\mu$ is the sum of the Milnor numbers of the singularities of $X_{\text {red }}$. Thus the multiplicity of the discriminant of plane conics at a double line is 2 , while for degree 3 and 4, the following 'kinds' of singular (resp., non-reduced) curves occur, with the indicated multiplicity (arrows denote 'specialization'):
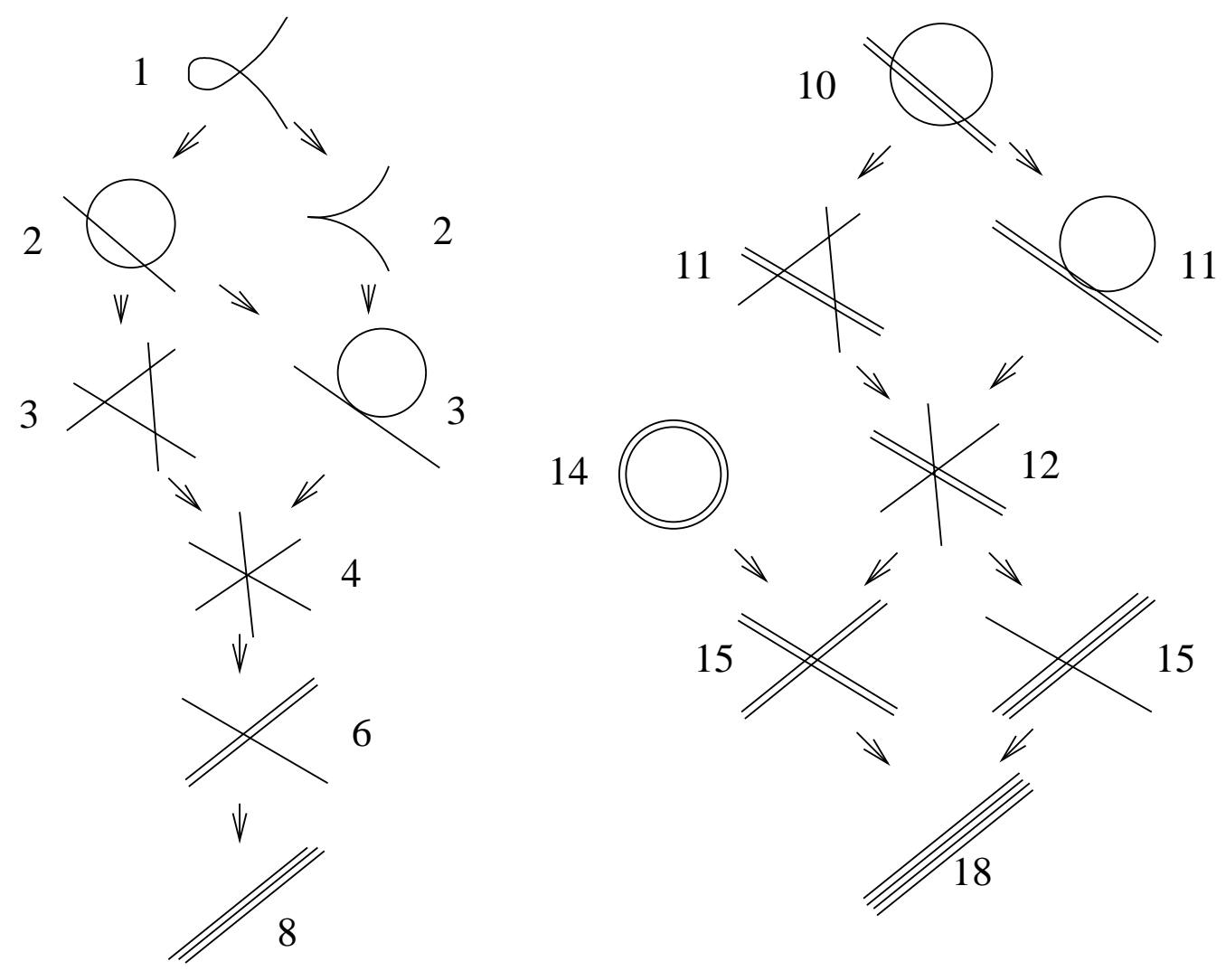

Example 3.2. For higher discriminants, let again $S=\mathbb{P}^{2}, \mathcal{L}=\mathcal{O}(d)$, and $V=$ $H^{0}\left(\mathbb{P}^{2}, \mathcal{O}(d)\right)$. For $0<i \leq d$ let $d^{(i)}=\operatorname{deg} X^{(i)}$; then Corollary 1.2 gives

$$
m_{X} D^{(i)}=\left[\left(\begin{array}{c}
i+2 \\
2
\end{array}\right)(d-i)-d^{(i)}\right] d^{(i)}+\mu_{i} .
$$

(Indeed $K$ has degree -3 , and the map $S \times H^{0}\left(\mathbb{P}^{2}, \mathcal{O}(d)\right) \rightarrow P^{i} \mathcal{O}(d)$ is clearly surjective for $i \leq d$. Also, it is clear that $\gamma_{i}=1$ in this case.)

For example, for $X$ a $d$-fold line one has $\mu_{i}=0$ for all $i>0$ (since $X_{\text {red }}$ is nonsingular), and $d^{(i)}=d-i$; so we get the multiplicity $m_{d, r}$ of the locus of degree- $d$ plane curves with a $\geq r$-tuple point, along the locus of $d$-fold lines, for $0<r \leq d$ : (let $i=r-1$ in the above)

$$
\begin{aligned}
m_{d, r} & =\left(\left(\begin{array}{c}
r+1 \\
2
\end{array}\right)-1\right)(d-r+1)^{2} \\
& =\frac{(r+2)(r-1)}{2}(d-r+1)^{2}
\end{aligned}
$$

In fact the computation runs just as easily to give the multiplicity $m_{n, d, r}$ of the locus degree- $d$ hypersurfaces in $\mathbb{P}^{n}$ with a $\geq r$-tuple point, along the locus of $d$-fold 
hyperplanes. First, for $S=\mathbb{P}^{n}$ the $\left(\begin{array}{c}n+i \\ n\end{array}\right)$ Chern roots of $\operatorname{Sym}^{i}(\Omega \oplus \mathcal{O})$ are all equal to $-i H$, where $H$ is the hyperplane class (indeed, the $n+1$ Chern roots of $\Omega \oplus \mathcal{O}$ are all equal to $-H$ since $\left.c(\Omega \oplus \mathcal{O})=(1-H)^{n+1}\right)$ so

$$
c\left(\operatorname{Sym}^{i}(\Omega \oplus \mathcal{O}) \otimes \mathcal{O}(d)\right)=(1+(d-i) H)^{\left(\begin{array}{c}
n+i \\
n
\end{array}\right)} ;
$$

with the same notation, $X^{(i)}=(d-i) H$; thus, applying the theorem gives

$$
\begin{aligned}
m_{n, d, r} & =\operatorname{deg}\left\{c\left(\operatorname{Sym}^{r-1}(\Omega \oplus \mathcal{O}) \otimes \mathcal{O}(d)\right) \cap s\left(X^{(r-1)}, \mathbb{P}^{n}\right)\right\}_{0} \\
& =\text { coeff. of } H^{n} \text { in }\left[(1+(d-r+1) H)^{\left(\begin{array}{c}
n+r-1 \\
n
\end{array}\right)} \frac{(d-r+1) H}{1+(d-r+1) H}\right] \\
& =(d-r+1)^{n} \cdot\left(\begin{array}{c}
n+r-1 \\
n \\
n-1
\end{array}\right)-1
\end{aligned}
$$

For $r=2$ this gives the well-known multiplicity $n(d-1)^{n}$ of a $d$-fold hyperplane in the ordinary discriminant. At the other end of the spectrum, we get the multiplicity of a $d$-fold hyperplane in the locus of degree- $d$ hypersurfaces with a $d$-tuple point (i.e., the cones from a point over a degree- $d$ hypersurface of $\mathbb{P}^{n-1}$ ):

$$
\left(\begin{array}{c}
n+d-1 \\
n \\
n-1
\end{array}\right)
$$

Example 3.3. To illustrate a case in which the singularities of $X_{\text {red }}$ are not isolated, consider $S=\mathbb{P}^{3}, \mathcal{L}=\mathcal{O}(d)$, and $X=$ union of three planes, with multiplicities $d_{1}, d_{2}, d_{3}$ adding up to $d$. We are going to compute the multiplicity $m_{X}$ of the ordinary discriminant at $X$. In this case $c((\Omega \oplus \mathcal{O}) \otimes \mathcal{O}(d))=(1+(d-1) H)^{4}$, where $H$ denotes the hyperplane class; so we only need to compute the Segre class of $J^{1} X$. One can distinguish several cases: in decreasing order of speciality (and thus necessarily with decreasing multiplicities)

-If $d_{2}=d_{3}=0$, then $X$ is simply a $d$-fold plane, so the previous example gives the multiplicity as

$$
3(d-1)^{3}
$$

-If $d_{1} \neq 0, d_{2} \neq 0, d_{3}=0$, then $X$ is the union of two distinct planes, say with equation $x^{d_{1}} y^{d_{2}}=0$. The Jacobian scheme $J^{1} X$ has ideal $\left(x^{d_{1}-1} y^{d_{2}}, x^{d_{1}} y^{d_{2}-1}\right)$, that is the divisor $x^{d_{1}-1} y^{d_{2}-1}=0$ with an embedded component along the line $(x, y)$ at which the planes intersect. The Segre class of $J^{1} X$ in $\mathbb{P}^{3}$ can then be obtained for example by applying [3], Prop. 9.2: the reader will check that $s\left(J^{1} X, \mathbb{P}^{3}\right)$ pushes forward to $\mathbb{P}^{3}$ to

$$
(d-2) H+\left(1-(d-2)^{2}\right) H^{2}+\left((d-2)^{3}-3(d-2)-2\right) H^{3}
$$

and applying the theorem yields

$$
m_{X}=(d-1)^{2}(3 d-4)
$$


-If $d_{1} \neq 0, d_{2} \neq 0, d_{3} \neq 0$ and the three (distinct) planes intersect along a common line, then the same procedure gives for $s\left(J^{1} X, \mathbb{P}^{3}\right)$

$$
(d-3) H+\left(4-(d-3)^{2}\right) H^{2}+\left((d-3)^{3}-12(d-3)-16\right) H^{3},
$$

from which

$$
m_{X}=(d-1)^{2}(3 d-5) .
$$

-Finally, if the three planes are in general position, say the equation for $X$ is $x^{d_{1}} y^{d_{2}} z^{d_{3}}$. Then $J^{1} X$ is the divisor $x^{d_{1}-1} y^{d_{2}-1} z^{d_{3}-1}$ with an embedded component along $(x y, x z, y z)$, supported along three 'coordinate' lines. To compute $s\left(J^{1} X, \mathbb{P}^{3}\right)$ one can blow-up $\mathbb{P}^{3}$ at the point common to the lines, then blow-up again along the proper transforms of the lines: the inverse image of $J^{1} X$ is a Cartier divisor in the top blow-up, and pushing forward the Segre class of this latter to $\mathbb{P}^{3}$ gives

$$
s\left(J^{1} X, \mathbb{P}^{3}\right)=(d-3) H+\left(3-(d-3)^{2}\right) H^{2}+\left((d-3)^{3}-9(d-3)-10\right) H^{3} \quad:
$$

from which, applying the theorem again, we get

$$
m_{X}=(d-2)(d-1)(3 d-2) \text {. }
$$

Example 3.4. Again let $S=\mathbb{P}^{3}$, and let $\mathcal{L}=\mathcal{O}\left(d_{1}+d_{2}\right)$. Let $X_{1}, X_{2}$ be smooth hypersurfaces of degrees $d_{1}, d_{2}$, intersecting along a curve $C$. If $X=X_{1} \cup X_{2}$, then $X$ is singular along $C$; the multiplicity $m_{X}$ of the discriminant at $X$ is then

$$
m_{X}=d_{1} d_{2}\left(3\left(d_{1}+d_{2}\right)-4\right) \quad .
$$

Indeed, in this case $J^{1} X=C$ is regularly embedded in $S=\mathbb{P}^{3}$, so $s\left(J^{1} X, S\right)=$ $c\left(N_{C} \mathbb{P}^{3}\right)^{-1} \cap[C]$ pushes forward to $\mathbb{P}^{3}$ to

$$
\frac{d_{1} d_{2} H^{2}}{\left(1+d_{1} H\right)\left(1+d_{2} H\right)}=d_{1} d_{2}\left(H^{2}-\left(d_{1}+d_{2}\right) H^{3}\right)
$$

(where again $H$ is the hyperplane class in $\left.\mathbb{P}^{3}\right)$, while $c((\Omega \oplus \mathcal{O}) \otimes \mathcal{O}(d))=(1+(d-$ 1) $H)^{4}:$ so

$$
m_{X}=\operatorname{deg}\left\{\left(1+4\left(d_{1}+d_{2}-1\right) H\right) \cdot d_{1} d_{2}\left(H^{2}-\left(d_{1}+d_{2}\right) H^{3}\right)\right\}_{0}
$$

with the above result. More generally, say a complete intersection curve $C$ is (scheme-theoretically) a connected component of the singular scheme of a degree- $d$ hypersurface $X$ in $\mathbb{P}^{n}$, and $\operatorname{deg} C=r, \operatorname{deg}\left(c_{1}\left(T_{C}\right)\right)=2-2 g$; then $C$ 'contributes' to $m_{X}$ by

$$
\operatorname{deg}\left\{(1+(d-1) H)^{n+1} \frac{r H^{n-1}+c_{1}\left(T_{C}\right)}{(1+H)^{n+1}}\right\}_{0}=r(d-2)(n+1)+2-2 g
$$

However, at least when $C$ is smooth there are strong constraints on what $r, d, n, g$ can actually be realized, so that for example the genus of the curve is determined by $r, d, n$. One can show that in this case the multiplicity will necessarily be

$$
\frac{r(4+(d-2)(n+3))}{2}
$$


These constraints can be derived by comparing the theorem in $\S 1$ to Parusiǹski's results $([\mathbf{1 2}])$. For example, a smooth curve of genus 2 cannot appear as the singular scheme of a hypersurface of $\mathbb{P}^{n}$. We will prove these facts elsewhere.

Example 3.5. Let $S=\mathbb{P}^{n_{1}} \times \cdots \times \mathbb{P}^{n_{r}}$, let $H_{1}, \ldots, H_{r}$ be the pull-backs of the hyperplane classes from the factors, and $\mathcal{L}=\mathcal{O}\left(H_{1}+\cdots+H_{r}\right)$ - that is, the bundle defining the Segre embedding of $S$. With this embedding, when is the dual variety of $S$ a hypersurface? The following criterion is proved in [5], $\S 3$, and can be deduced from more general criteria in [10] (see [5], Theorem 1.3 and Lemma 3.5). We give here a simple direct argument.

Proposition 3.1. The dual variety of $S=\mathbb{P}^{n_{1}} \times \cdots \times \mathbb{P}^{n_{r}}$ is a hypersurface if and only if $2 n_{i} \leq n=\sum_{j} n_{j}$ for all $i$.

Proof: Let $x^{i}=\left(x_{0}^{i}, \ldots, x_{n_{i}}^{i}\right)$ denote homogeneous coordinates on $\mathbb{P}^{n_{i}}$. If $h \in$ $H^{0}(S, \mathcal{L})$ then $h$ is a multilinear function in $x^{1}, \ldots, x^{r}$, and $h$ is a singular section if and only if the system of equations

$$
\frac{\partial h}{\partial x_{j}^{i}}=0 \quad\left(1 \leq i \leq r, 0 \leq j \leq n_{i}\right)
$$

has a non-trivial solution (i.e. a solution with $x^{i} \neq 0$ for all $i$ ).

Suppose that the condition $2 n_{i} \leq n$ for all $i$ is not satisfied; for simplicity of notation let us assume that it fails for $i=1$, so that $n_{1}>m_{1}=\sum_{j>1} n_{j}$. Let $h \in H^{0}(S, \mathcal{L})$ denote any singular section. We claim that the singular locus of $h$ is positive-dimensional, and hence the dual of $S$ is small. In fact, let $x=\left(x^{1}, x^{2}, \ldots, x^{r}\right)$ denote a singular point of $h$; it is easy to see from the system of equations above that the singular points of $h$ of the form $\left(y^{1}, x^{2}, \ldots, x^{r}\right), y^{1} \in \mathbb{P}^{n_{1}}$, form a family of dimension $n_{1}-m_{1}$.

Conversely, suppose $2 n_{i} \leq n$ for all $i$. We prove by induction on $r$ that there exist sections $h$ with isolated singularities. The initial case $r=2$ is easy; for $r>2$, arrange the indices so that $n_{i} \geq n_{i+1}$ for all $i$, let $S^{\prime}=\mathbb{P}^{n_{2}} \times \cdots \times \mathbb{P}^{n_{r}}$, and $\mathcal{L}^{\prime}=\mathcal{O}\left(H_{2}+\cdots+H_{r}\right)$. We claim that there exist $h_{0} \in H^{0}\left(S^{\prime}, \mathcal{L}^{\prime}\right)$ with singularity locus of dimension at most $n_{1}$ (notice that $n_{1} \leq m_{1}=\operatorname{dim}\left(S^{\prime}\right)$ ). To see this, we treat two separate cases: first, if $n_{2} \leq N=\sum_{j>2} n_{j}$ then by the inductive hypothesis there exists $h_{0} \in H^{0}\left(S^{\prime}, \mathcal{L}^{\prime}\right)$ with isolated singularities; second, if $n_{2}>N$ take $h_{0}=\sum_{0 \leq j \leq N} x_{j}^{2} f_{j}\left(x^{3}, \ldots, x^{r}\right)$ where the $f_{j}$ are general multilinear forms in the indicated variables. It is easy to see (as in the argument above) that the singular locus of $h_{0}$ has dimension $n_{2}-N<n_{1}$. Choose then such an $h_{0}$ and choose non-singular sections $h_{1}, \ldots, h_{n_{1}} \in H^{0}\left(S^{\prime}, \mathcal{L}^{\prime}\right)$ so that the set $h_{1}=\cdots=h_{n_{1}}=0$ intersects the singular locus of $h_{0}$ in isolated points (since $\mathcal{L}^{\prime}$ is very ample, this can be achieved). It is now easy to check that $h=\sum x_{i}^{1} h_{i}$ has isolated singularities.

It is natural to try to use Proposition 1.3 to prove (3.1). Curiously, if one uses (3) in Prop. 1.3 (or equivalently [3], p. 63), the combinatorics becomes rather involved; but it is easy to show that the dual of $S$ is small if the numerical conditions are not satisfied, using Prop. 1.3 (2). By just choosing one singular section and showing that the class in (2) is 0 , one shows that all singular sections must have positive dimensional singular locus. 
So we need to produce a singular divisor $X$ on $S$ such that

$$
\operatorname{deg}\left\{c((\Omega \oplus \mathcal{O}) \otimes \mathcal{L}) \cap s\left(J^{1} X, S\right)\right\}_{0}=0 \quad ;
$$

the trick is to choose $X$ so that $J^{1} X$ is 'contained in one factor' of the product, so that the computation of the Chern class becomes manageable.

Using the above notation, $n_{1}>m_{1}$. Let $\left(x_{0}: \cdots: x_{n_{1}}\right)$ be coordinates in $\mathbb{P}^{n_{1}}$, and choose $m_{1}$ sections $h_{i} \in H^{0}\left(S^{\prime}, \mathcal{L}^{\prime}\right)$ for $0 \leq i<m_{1}$ that are non-singular and intersect transversally. Then let $X$ be the divisor on $S$ defined by $h=\sum_{0 \leq i<m_{1}} x_{i} h_{i}$ : $J^{1} X$ has ideal $\left(h_{0}, \ldots, h_{m_{1}-1}, x_{0}, \ldots, x_{m_{1}-1}\right)$-that is, it consists of the disjoint union of several spaces $\mathbb{P}^{n_{1}-m_{1}}=\mathbb{P}^{n_{1}-m_{1}} \times\left\{\left(p_{2}, \ldots, p_{r}\right)\right\}$, as $\left(p_{2}, \ldots, p_{r}\right)$ runs through the list of the points of intersection of $h_{0}, \ldots, h_{m_{1}-1}$. It's enough then to show that the above degree is 0 for each of these components. Now notice that $H_{2}, \ldots, H_{r}$ are trivial on each component, so with obvious notations

$$
\begin{aligned}
& \left\{c((\Omega \oplus \mathcal{O}) \otimes \mathcal{L}) \cap s\left(\mathbb{P}^{n_{1}-m_{1}}, S\right)\right\}_{0} \\
= & \left\{c\left(\left(\Omega_{\mathbb{P}^{n_{1}}} \oplus \mathcal{O}^{m_{1}+1}\right) \otimes \mathcal{O}\left(H_{1}\right)\right) \cap \frac{\left[\mathbb{P}^{n_{1}-m_{1}}\right]}{\left(1+H_{1}\right)^{m_{1}}}\right\}_{0} \\
= & \left\{\left(1+H_{1}\right)^{m_{1}} \frac{\left[\mathbb{P}^{n_{1}-m_{1}}\right]}{\left(1+H_{1}\right)^{m_{1}}}\right\}_{0} \\
= & \left\{\left[\mathbb{P}^{n_{1}-m_{1}}\right]\right\}_{0}=0
\end{aligned}
$$

since $n_{1}>m_{1}$.

\section{REFERENCES}

[1] A. Dimca, Milnor numbers and multiplicities of dual varieties, Rev. Roumaine Math. Pures et Appl. 31 (1986), 535-538.

[2] L. Ein, Varieties with small dual varieties, Inventiones Mathematicae 86 (1986), 63-74.

[3] W. Fulton, "Intersection Theory," Springer Verlag, 1984.

[4] W. Fulton, S. Kleiman, R. Piene, H. Tai, Some intrinsic and extrinsic characterizations of the projective space, Bull. Soc. math. France 113 (1985), 205-210.

[5] I. M. Gelfand, M. M. Kapranov and A. V. Zelevinsky, "Hyperdeterminants," Cornell University, Technical Report no. 26, 1991.

[6] M. Greenberg, "Lectures on Algebraic Topology," Benjamin, 1967.

[7] P. Griffiths and J. Harris, "Principles of Algebraic Geometry," John Wiley \& Sons, 1978.

[8] A. Grothendieck, EGA IV (4), Publ. Math. IHES, no. 32 (1967).

[9] S. Kleiman, Tangency and Duality, Can. Math. Soc. Conf. Proc. 6 (1986).

[10] F. Knop, G. Menzel, Duale Varietäten von Fahnenvarietäten, Comm. Math. Helv 62 (1987), 38-61.

[11] A. Némethi, Lefschetz theory for complex affine varieties, Rev. Roumaine Math. Pures et Appl. 33 (1988), 233-250.

[12] A. Parusiǹski, Multiplicity of the dual variety, Bull. of the London Math. Soc. 23 (1991).

[13] R. Piene, Numerical characters of a curve in projective n-space, "Real and complex singularities," Sijthoff and Noordhoff, 1977, pp. 475-495.

Mathematics Department, Florida State University, Tallahassee FL 32306

Mathematics Department, University of Kansas, Lawrence KS 66045 